Magnus Ronn | LM Tucker | Ron Dulaney Jr. Sinclair, Mousazadeh, Safarzadeh | James Shraiky Marci Uihlein | Tang, Anderson, Aksamija, Hodge

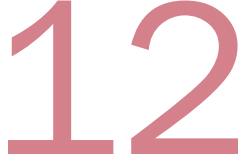

volume 9 | issue 1

\title{
Performative Computation-aided Design Optimization
}

\author{
Ming Tang, University of Cincinnati \\ Jonathon Anderson, University of North Carolina Greensboro \\ Ajla Aksamija, PhD, Perkins+Will \\ Michael Hodge, Perkins+Will
}

\section{Introduction}

This article discusses a collaborative research and teaching project between the University of Cincinnati, Perkins+Will's Tech Lab, and the University of North Carolina Greensboro. The primary investigation focuses on the simulation, optimization, and generation of architectural designs using performance-based computational design approaches. The projects examine various design methods, including relationships between building form, performance and the use of proprietary software tools for parametric design.

\section{Current Issues for Form Seeking}

As a result of the extensive development of CAD / CAM technologies, architects have achieved an unprecedented level of freedom to model and fabricate forms. The computational design software combined with parametric modeling tools allows designers to directly manipulate freeform models, but without considering the performance analysis. Most form seeking methods include various algorithms and scripting processes, which can generate a large number of iterations and allow architects to "freeze" the form configuration at certain stage based on their own judgment. However, this process is primarily based on form's visual properties. Grobman and Neuman described it as a design method focusing "mainly to the outcome of visual properties, on image, while neglecting to incorporate other aspects of architecture" (2012). As a result, "odd forms" are generated and often not able to be realized in the physical landscape.

The authors generally define building performance as an expression of measurable variables which affect a process or procedure. Common building performance factors are environmental factors, such as solar gain, aerodynamics, and heat loss, structural factors such as load and stress, and social factors such as view and privacy. In the proposed design process, performance factors are critical parameters that drive the building form and ultimately the construction process.

The disconnection between "visual-driven form seeking" methods and the "performance driven design " principles intensified with the rapid development of digital fabrication. The freedom in visual-driven form making can easily lead to disregarding its function, material properties, and still be able to be constructed physically while neglecting the buildings' performance. There is a truly innovative design process that exists in using performance-based input parameters to generate forms that are not only aesthetically pleasing but also sustainable in nature . (Aksamija and Mallasi, 2010; Aksamija et al., 2011).

\section{Research Methodology}

Realizing the importance of performance driven design provokes a question that is vital to the field of architectural design: what is the
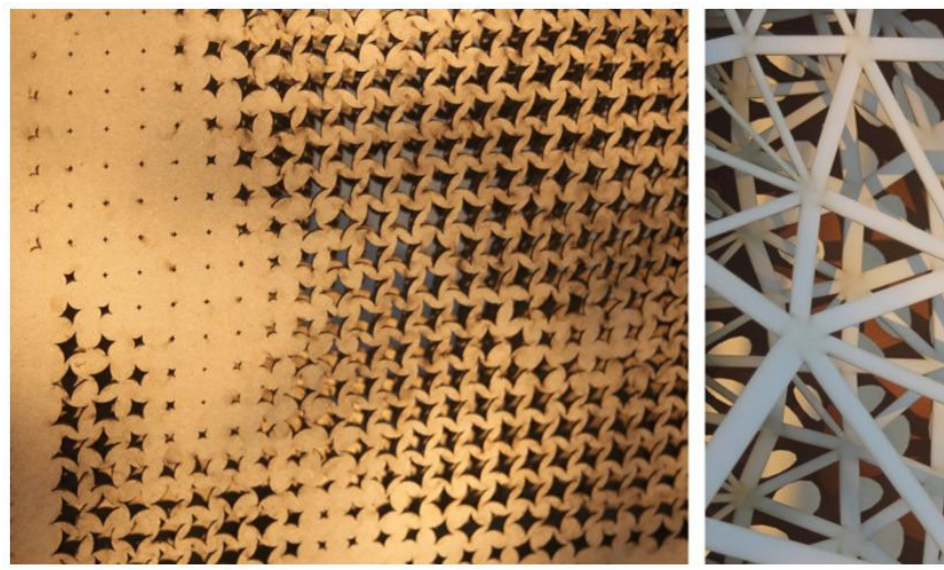

Figure 1. These pattern making projects investigate how the large quantity of iterations can be filtered and selected based on the feasibility and maximization of day light. The parameters used in this instance relate to armature, smart skin, and were driven by environmental data in order to promote heterogeneity.
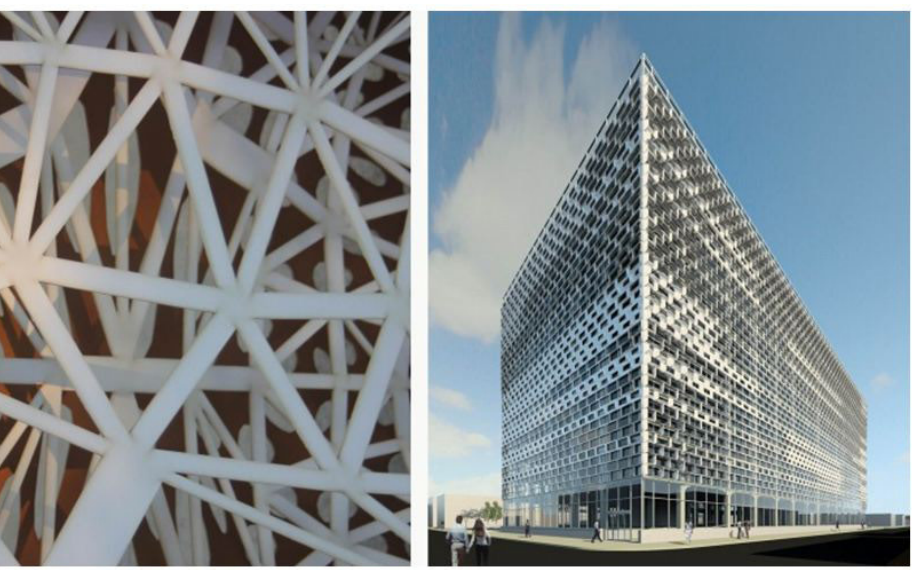

Patterns are created based on mass customization and a direct performative exchange with a specific environment. Students: Andy McCarthy, Jessica Helmer, Dylan Fischer. University of Cincinnati. 
relationship between form and performance? Grobman and Neuman described how the performance should go beyond just the functionalist utilitarian aspects, (which) "is not necessarily addressing the complexity of form as culture, social and political product" (2012). How can we apply performance driven design as an optimization process, which is not only answering the building needs in terms of technical details but also providing a form that speaks a desired language?

To answer these questions, several design courses have been developed by faculty, researchers, and practitioners at the University of Cincinnati, Perkins+Will, and University of North Carolina Greensboro. The authors used several different strategies to investigate both the technical optimization (rational process) and human optimization (intuitive process). By utilizing CAD/CAM, simulations, and fabrication tools the authors realized the marriage of computation and prescriptive form seeking through an interdisciplinary collaboration that relies on quantitative data.

\subsection{Technical Optimization}

Performance-based design relies on evaluation and performance simulations by using highly technical computer programs that are often complex. In 2010, a graduate course titled "performance driven design and non-linear thinking" explored the generation of forms by regulating a series of parameters and their relations to another in the context of parametric correlations. In these investigations, functions related to a single parameter; such as wind, sun, or stability of the construction technique (Figure 1). Students discovered how numerically controlled environments affect the overall performance quality and character of an initial artifact. This process adapted and was referred as the "shared body
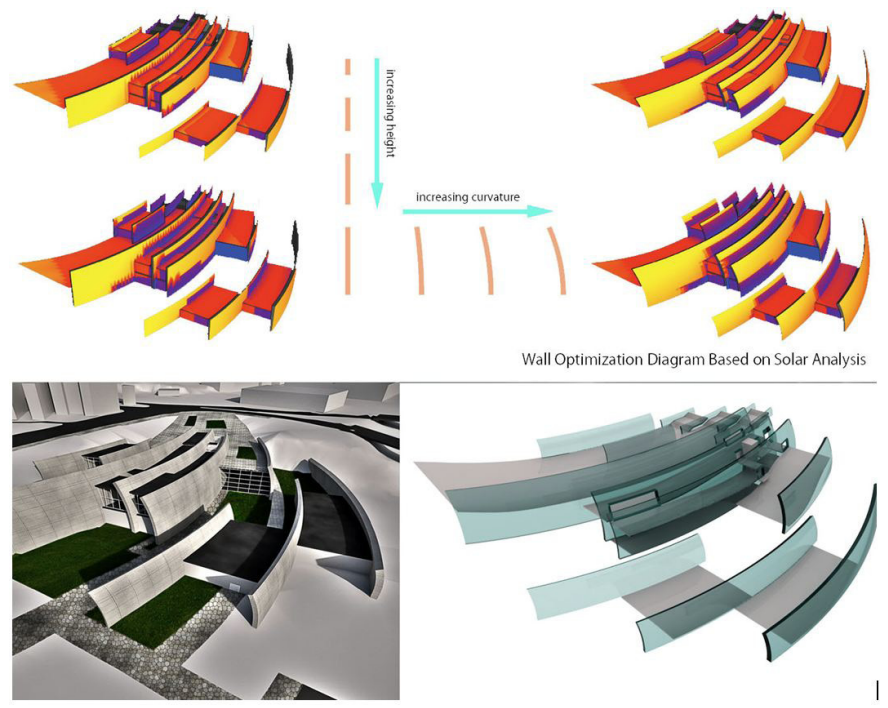

Figure 2. Design and optimization of wall height and curvature based on Ecotect solar access analysis. Tools: Rhino, Grasshopper, Geco, Ecotect.

Student: Stephan Dober, University of Cincinnati.

plan ". Design methodologies, such as the use of building performance and simulation tools, genetic morphing, and fitness evaluations were discussed as new paradigms in performance-based design. Digital tools such as Maya, Rhino, Grasshopper, Vasari, and Ecotect were introduced to the process as a means to simulate, analyze environments, and seek form. For instance, a parametric wall system supported by instant feedback from Ecotect analysis can be used to optimize the wall profile (Figure 2).
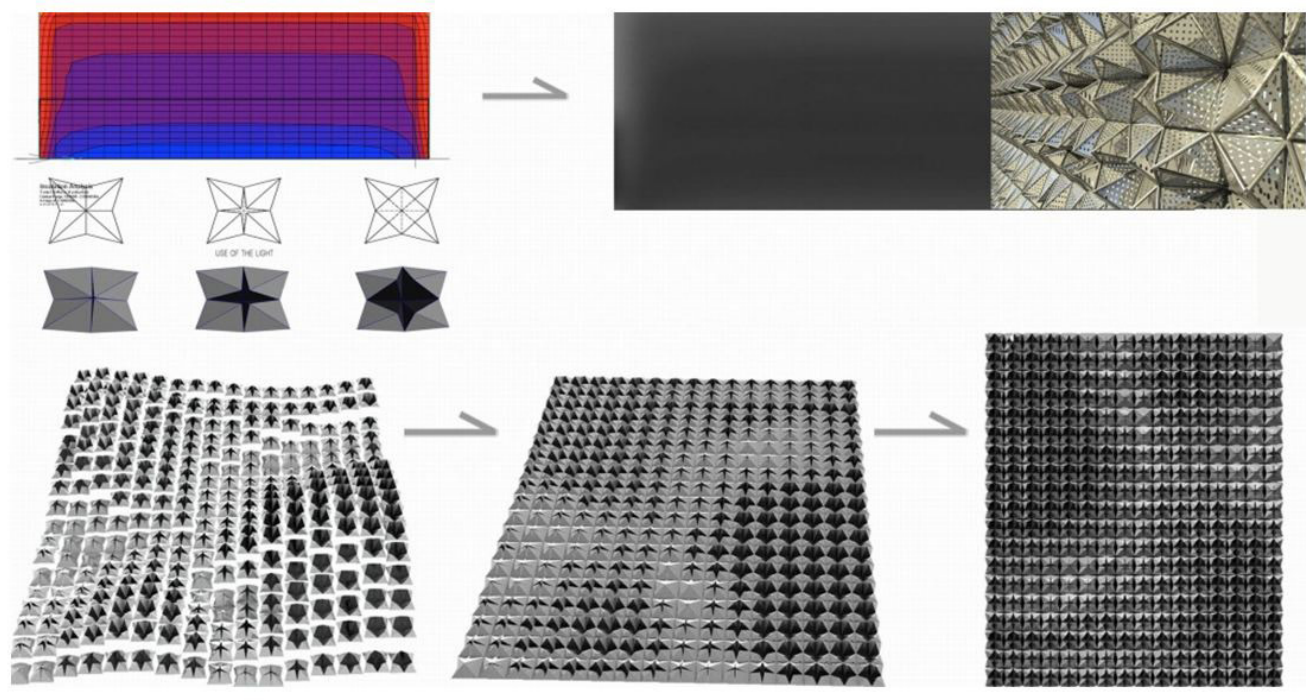

Figure 3: Origami patterns are generated by parameters that correspond to the impact of the sun on the facade, wind and rainwater. Student: Mailys Meyer. University of Cincinnati. 

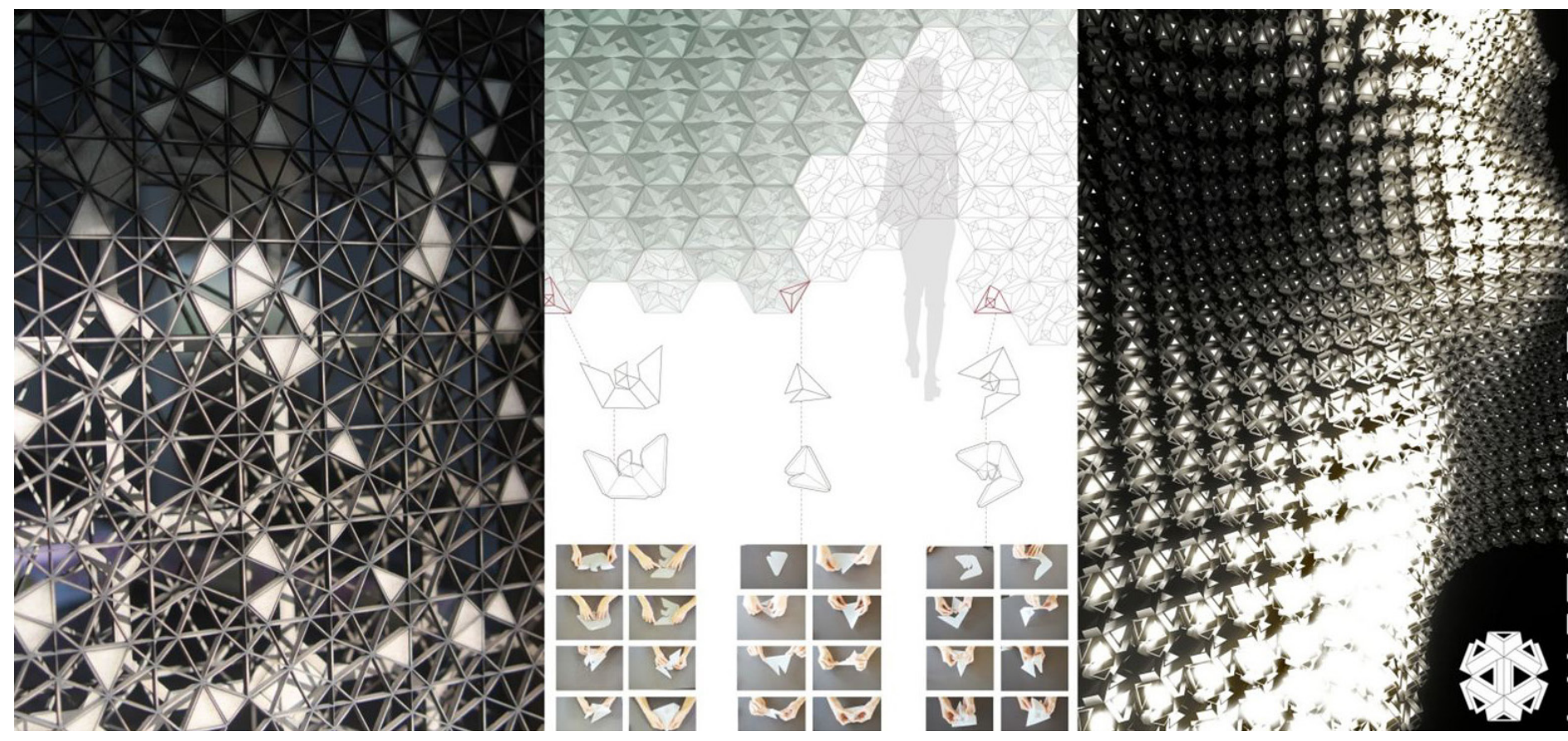

Figure 4: Investigation of the connection between craft and computation. Hexagon patterns have been implemented to generate a large quantity of creative solutions, whereas origami folds, or tabs, are introduced as material optimizers. Left: Screen Design. Natalie Levinson. University of Cincinnati. Middle: "ephemeral membrane" a second year collaborative project at the University of North Carolina Greensboro. Right: Light design. Derek Sommers, Frederik Berte, University of Cincinnati.

\subsection{Human Optimization: Integration of visual-driven and performance driven process}

In the human optimization approach, the performance parameters are social or cultural values instead of structural and environmental factors. For the purpose of this research, the use of origami folding was introduced as an example and discussed in terms of social and cultural elements that apply to performance based form-seeking methods. For example, a student's project could use technical data and complex systems to respond to solar radiation and drive an armature's open/close operations, but the human optimization looked to create a unique cultural value in terms of patterning or solid/void relationships (Figure 3). Here, both visual driven and utilitarian parameters drive the evolution of the armature's "shared body plan".

This hybrid method investigates how a large quantity of iterations can be filtered and selected based on the feasibility of fabrication and materialization as it relates to human perception. This process is viewed as an investigation of varying scales, creation of hierarchy in prototypes, and a platform for understanding the performance of parts in the context of the whole. Human optimization shifts utilitarian driven process to a complex dynamic operation based on performative aspects. The response to the social and cultural parameters, craftsmanship, and material issues are used to manually modify design solution to adapt to these constraints - an analog exploration of performance driven design (Figures 4). With this approach, we tried to find a middle ground between the perceptual image and functionalist attitude toward architectural form.

\section{Projects}

Over the past year, several courses have been introduced for teaching performance driven processes. The authors analyzed several design and prototyping procedures, and illustrate how these performance-driven methods are used for innovative design approaches and outcomes, employing benefits of performance-based influences in architecture beyond aesthetic experimentation.

\subsection{Project: Anthropometric Garments}

This exercise introduced students to observing and documenting the human body through anthropometric and ergonomic analyses. Within this framework each student learned to develop techniques on how to measure their body, log data, and make use of such information to construct a story about their own findings, discoveries, techniques, and process.

Designers need to seek further understandings of these fundamental changes/shifts in scales, proportions, and hard data in order to gain insightful methods that can potentially advance the conversations already spearheaded by previous scholars. These investigations generate 

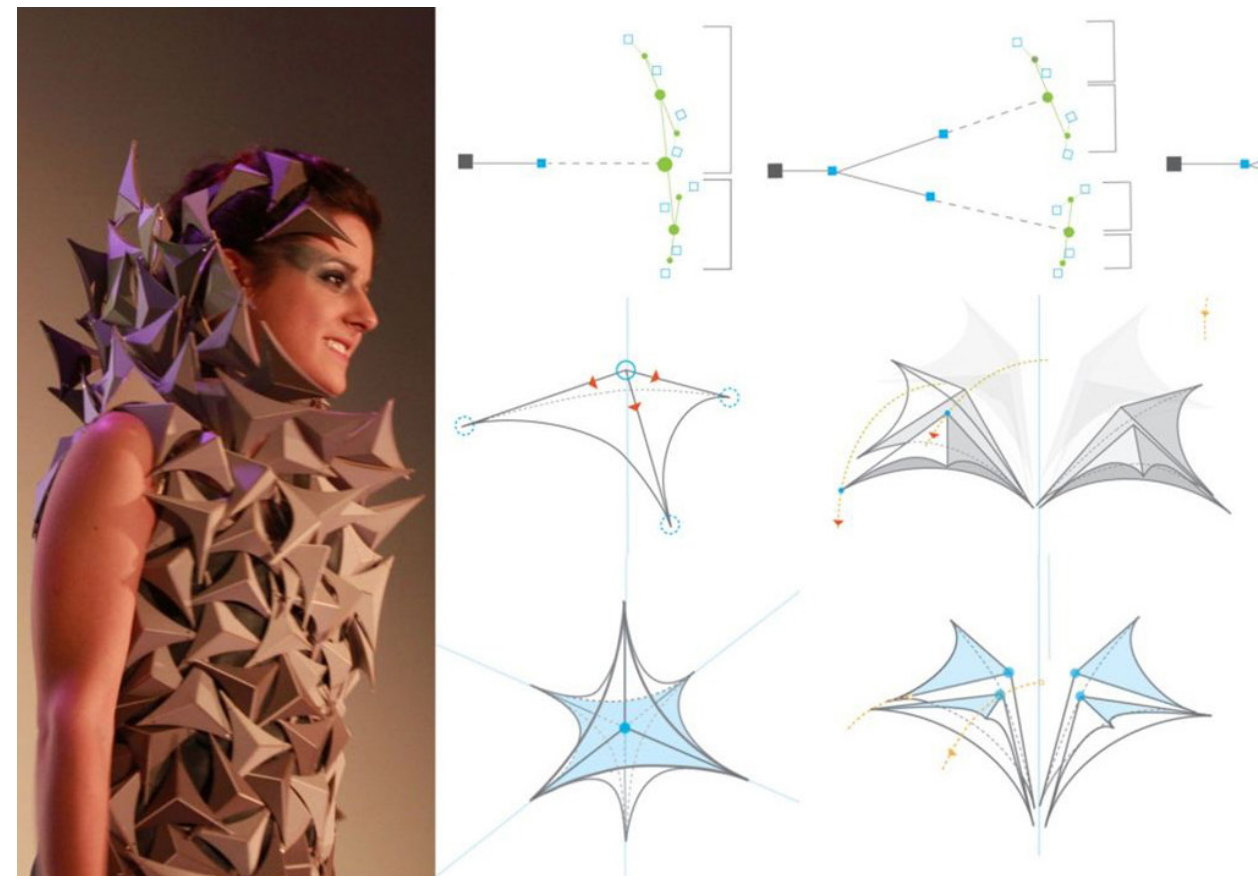

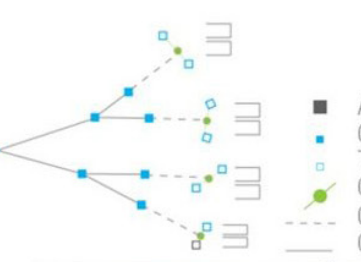

ANCHOR GROWN POINT TARGETED POINTS CENTROID OF PAIR GROWTH VECTOR CONNECTING STRUCTURE

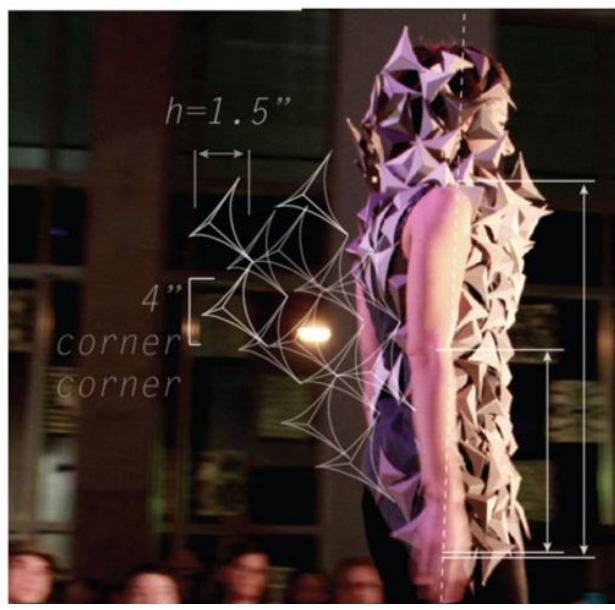

Figure 5. A student's design of a garment that is generated and responds to human performance and ergonomic data. Work of Laura Kimmel, second year interior architecture student at the University of North Carolina Greensboro

relationships and use visual and performative data, which is extracted from the human body, to ultimately drive form. For example, a garment was generated based on anatomically informed units that regenerate away from the body's central axis and develop branched patterns that respond to the body's subdivisions, proportions, and organization (Figure 5). Architects must be able to gain a greater understanding of their own body in order to design objects that are conscious of the human experience and use the body as the generator of data that results in a "shared body plan".

\subsection{Project 2: Scuta - Re: Skin}

"Scuta" is a student project which investigates the relationship between performance based and visual-based design. The objective of the studio was to promote and assist students to experiment with a performancebased design approaches in order to design a building skin. The student "deconstructed" the building envelope to components that make up the shell or skin of the building. The envelope responds to performative values, such as climate, ventilation, and energy consumption within the structure. Furthermore, it also provides an aesthetic statement which has symbolic references to that of an animal's scales (Figure 6).

The student designed a few variations stemming from the idea of the scuta. With customized tools written in Maya MEL and Revit API 4, the student used a bottom to top approach to design the paneling system. Starting with the issues of protection and ideas for fabrication, the component sponsored the process of building the system and the final assembling.

Using Ecotect as a simulation tool to capture solar radiation values, the student was able to create a "shared body plan" that output a blended surface in Maya. Each of the building component's aperture used sun azimuth angles to drive the forms scale, openness and ultimately the aesthetic quality. On the south side of the building the components featured a larger extrusion at the top of the window to shade from harsh sunlight and reduce glare. On the east and west sides, the components have a larger extrusion on the left and right side.

To prototype the component, the student designed a system that could be formed depending on the parameters of the system. The student milled a component for each polar extremity out of high density foam and vacuum-formed the units out of polystyrene. The project demonstrated how performance driven design should simultaneously address multiple constraints including environmental, structural aspect, perceptual, behavioral aspects, as well as the associative material parameters .

\section{Conclusion}

The relationship between form, meaning, and performance generates a new design methodologies and language. This research intends to show an inventive approach to merging these new ways of thinking with the basic values that we expect to see as a generator of design products. 


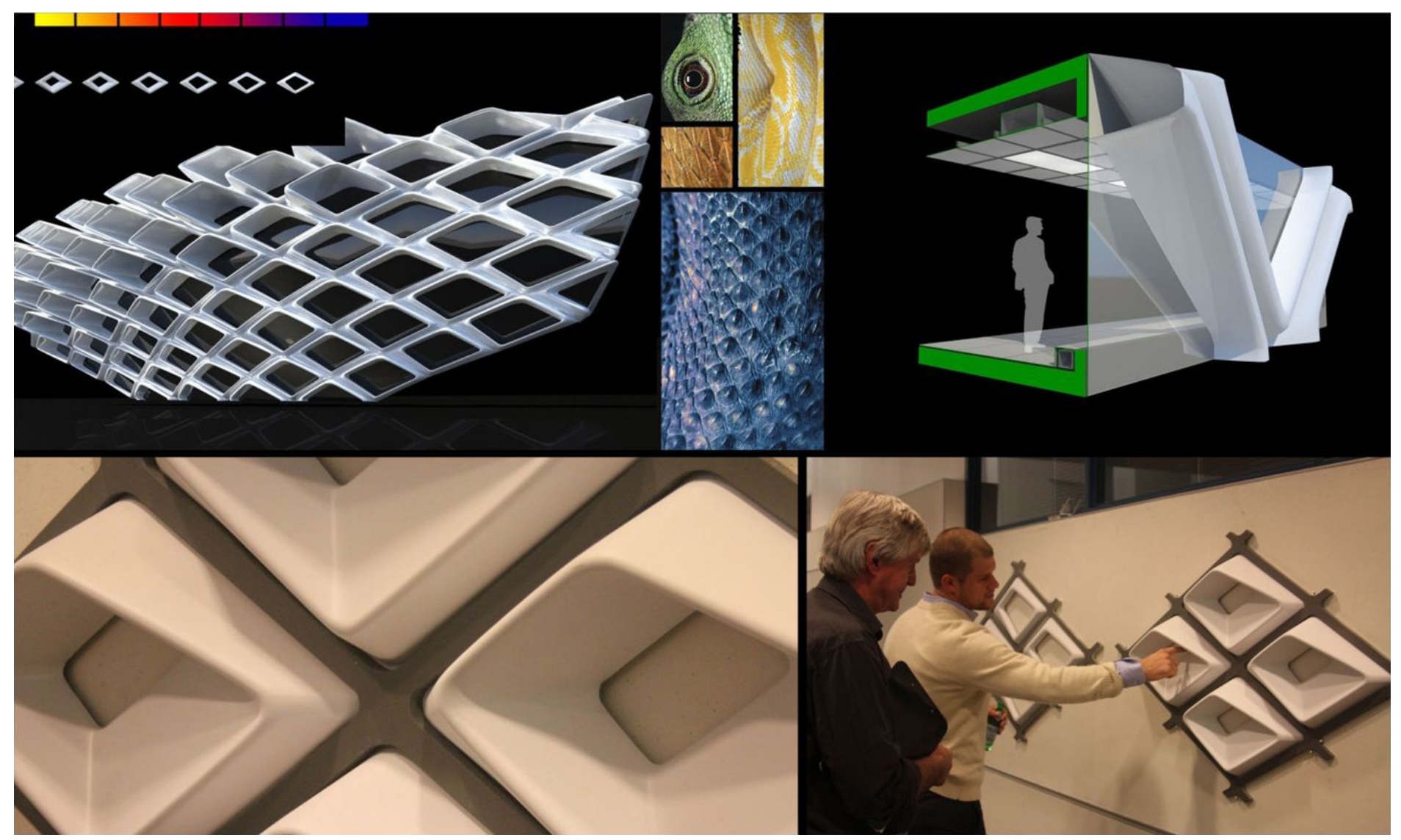

Figure 6. Using natural influences such as the scales of reptile skin served as a primary influence for the concept analysis. Scuta (latin scutum, meaning "shield") is the bony external plate or scale, as on the shell of a turtle, skin of crocodilians, and some feet of birds. The concept of the Scuta was intriguing not only because of its function, but more importantly for its geometrical divergence. For example, the parameters of each scale on the alligator changes depending on its location on the body. This metaphor provides an interesting way of looking at the façade of a building. The metaphor can be applied on a variety of scales, from site to the prototypical window components. By student Drew Newman.

Through this investigation we gained a greater understanding of the process necessary in the creation of a dynamic system, which is not based on its visual/aesthetic properties but instead on the performative factors that influence our landscape.

As a simplified approximation of a complex system, both physical and virtual prototyping were used to study the form finding. The physical prototyping allowed students to investigate material properties and structural behaviors in either full scale or scaled mock-ups. We believe performance driven design should work with visual-driven design in order to obtain architecture that is conscious and responds to various parameters. This ongoing investigation will continue to develop and increase our understanding of parametric design as it relates to performative capabilities.

1. Supported by the modeling and simulations, Performance Based Design (PBD) uses evidence and data as the essential design driver, rather than architect's intuition. It is a process to create a system of parameters which can be verified, validated and evaluated with facts. Oxman described it as design informed by internal evaluation. Oxman describes this approach as "a determinant and method for the creation of architectural form. In such circumstances digital design diverges from a design paradigm in which the formal manipulative skills and preferences of the human designer externally control the process to one in which the design is informed by internal evaluative and simulation processes." (Oxman, 2009)

2. Compared with traditional design methods, Building performancebased design method: has power in predicting a design solution because it: (1) uses performance measures with actual quantifiable data and not rules-of-thumb; (2) aims to develop a model of a complex physical system; (3) uses the model to analyze and predict behavior of the system; and (4) produces a more realistic evaluation of the design. (Aksamija and Mallasi, 2010; Aksamija et al., 2011).

3. "Shared body plan" can be defined by common ancestors found in natural life forms passing their shared body to the offspring. In other words, by changing the proportions of the components in the shared body plan, various types of creatures begin to be generated. As described by De Landa, "a kind of 'abstract vertebrate' which, if folded and curled in particular sequences during embryogenesis, yields an elephant, twisted and stretched in another sequence yields a giraffe, and in yet other sequences of intensive operations yields snakes, eagles, sharks and humans." Manuel later adds that "... if evolved architectural structures are to enjoy the same degree of combinatorial productivity as biological ones 
they must also begin with an adequate diagram, an abstract building corresponding to the abstract vertebrate". (De Landa, 2001).

4. "White Feet" Revit plugin developed by Perkins + Will was used to size and position building skin components, and determine appropriate forms and geometry based on performance analysis results from Ecotect and other simulation programs.

5. At the end of the investigation, the student commented on the difference of this new performance driven approach compared to conventional image-driven method: "The scale of each part in the design scheme matches an underlying knowledge of proportion. In every step of its process from computation to fabrication, the evolution of its proportion was well conceived as its design goal. This part-to-whole relationship has been a constant aesthetic problem of parametric design that I believe was answered with this approach. Without the issues of scale and proportion the meaning and aesthetics can be led astray, leaving any number of possible solutions unresolved. With this proposal, I believe the questions that have always been prevalent in design theory are answered." By student Drew Newman, University of Cincinnati.

6. "(Because) integrating material properties values into a digital evaluation system is very difficult. That is the reason the prototyping with fabrication techniques and testing various post-digital actions is important part of the generative design process." ( Tang et al., 2012)

\section{References}

Aksamija, A., Guttman, M., Rangarajan, H., and Meador, T., 2011. "Parametric Control of BIM Elements for Sustainable Design in Revit: Linking Design and Analytical Software Applications through Customization", Perkins+Will Research Journal, Vol. 3, No. 1, pp. 32-45. Aksamija, A., and Mallasi, Z., 2010. "Building Performance Predictions: How Simulations Can Improve Design Decisions", Perkins+Will Research Journal, Vol. 2, No. 2, pp. 7-32.

De Landa, M., 2001. "Deleuze and the Use of the Genetic Algorithm in Architecture", Design for a Digital World, New York, NY: Wiley, pp. 117120.

Grobman, Y., and Neuman, E., 2012. Performalism: Form and Performance in Digital Architecture, New York, NY: Routledge, pp. 1-8.

Oxman, R., 2009. "Performance-based Design: Current Practices and Research Issues", International Journal of Architectural Computing, Vol. 6, No. 1, pp. 1-17.

Tang, M., Anderson, J., Aksamija, A., and Hodge, M., 2012. "PerformanceBased Generative Design, An investigation of the Parametric Nature of Architecture.", Proceedings of the 100th ACSA Conference: Digital Aptitudes, Boston, MA.

\section{Endnotes}

1. Supported by the modeling and simulations, Performance Based Design (PBD) uses evidence and data as the essential design driver, rather than architect's intuition. It is a process to create a system of parameters which can be verified, validated and evaluated with facts. Oxman described it as design informed by internal evaluation. Oxman describes this approach as "a determinant and method for the creation of architectural form. In such circumstances digital design diverges from a design paradigm in which the formal manipulative skills and preferences of the human designer externally control the process to one in which the design is informed by internal evaluative and simulation processes." (Oxman, 2009)

2. Compared with traditional design methods, Building performancebased design method: has power in predicting a design solution because it: (1) uses performance measures with actual quantifiable data and not rules-of-thumb; (2) aims to develop a model of a complex physical system; (3) uses the model to analyze and predict behavior of the system; and (4) produces a more realistic evaluation of the design. (Aksamija and Mallasi, 2010; Aksamija et al., 2011).

3. "Shared body plan" can be defined by common ancestors found in natural life forms passing their shared body to the offspring. In other words, by changing the proportions of the components in the shared body plan, various types of creatures begin to be generated. As described by De Landa, "a kind of 'abstract vertebrate' which, if folded and curled in particular sequences during embryogenesis, yields an elephant, twisted and stretched in another sequence yields a giraffe, and in yet other sequences of intensive operations yields snakes, eagles, sharks and humans." Manuel later adds that "... if evolved architectural structures are to enjoy the same degree of combinatorial productivity as biological ones they must also begin with an adequate diagram, an abstract building corresponding to the abstract vertebrate". (De Landa, 2001).

4. "White Feet" Revit plugin developed by Perkins + Will was used to size and position building skin components, and determine appropriate forms and geometry based on performance analysis results from Ecotect and other simulation programs.

5. At the end of the investigation, the student commented on the difference of this new performance driven approach compared to conventional image-driven method: "The scale of each part in the design scheme matches an underlying knowledge of proportion. In every step of its process from computation to fabrication, the evolution of its proportion was well conceived as its design goal. This part-to-whole relationship has been a constant aesthetic problem of parametric design that I believe was answered with this approach. Without the issues of scale and proportion the meaning and aesthetics can be led astray, leaving any number of possible solutions unresolved. With this proposal, I believe the questions that have always been prevalent in design theory are answered." By student Drew Newman, University of Cincinnati.

6. "(Because) integrating material properties values into a digital evaluation system is very difficult. That is the reason the prototyping with fabrication techniques and testing various post-digital actions is important part of the generative design process." ( Tang et al., 2012). 